\title{
Feature extraction and influence analysis for the structural performance of complex component
}

\author{
Haiyan Hua ${ }^{1, *}$, Zhenhui Shen ${ }^{2}$, and Yangmei Zhang ${ }^{2}$ \\ ${ }^{1}$ School of Mechanical \& Automotive Engineering, FuJian University of Technology, Fuzhou 350118, P.R. China \\ ${ }^{2}$ Engineering College, Fujian Jiangxia University, Fuzhou 350108, P.R. China
}

\begin{abstract}
To explore the structural performance of complex component deeply, a new method of feature extraction and influence analysis is proposed and its automatic mechanism is established. Using this mechanism, the complex component can be divided into several sub-regions, and the maximum stress in each sub-region can be extracted. After evaluating the danger situation for all sub-regions, the absolute influence of structural parameters for the characteristic stresses in feature regions are carried out based on Spearman rank correlation analysis. And then, the feature knowledge of complex component is acquired. Finally, the excavator boom is taken as an example, which demonstrates that the useful knowledge can be extracted from the structural performance of complex component automatically and effectively by this method.
\end{abstract}

Keywords. Feature extraction; Influence analysis; Structural performance; Spearman rank correlation coefficient; Significant sensitive

\section{Introduction}

High strength and lightweight are two important but contradictory targets for modern mechanical design[1], which are more difficult to be coordinated for complex component. Exploring the stress feature is one of the most important work for realizing reasonable lightweight design and improving the comprehensive structural performance of complex component [2,3]. In recent years, many feasible strategies of extracting stress features have been proposed in some researches. For example, the welding regions have been taken as stress features by Uzer[4], but the influence of complex geometry for the structure performance is still not be solved. The regions of the bus with different shapes and sizes have been explored and optimization by Zhang, but the curved beam of the bus needs to be simplified to straight beam[5]. However, using existing method, the stress features of complex component cannot be fully extracted because of the continuous change of geometry. And the influence for stress features is also difficult to be acquired. For complex component, it is necessary to seek a more effective method to extract the stress feature.

Therefore, a new method of automatic extracting stress features based on stress survey is proposed in this paper. And then, the influence analysis for stress features based on Spearman rank correlation analysis is discussed.

\section{An automatic mechanism for feature extraction and influence analysis}

To explore the structural performance of complex component deeply, an automatic mechanism for feature extraction and influence analysis (FE\&IA Mechanism) is constructed and shown in Fig.1. This mechanism includes three major modules, which are illustrated as follows.

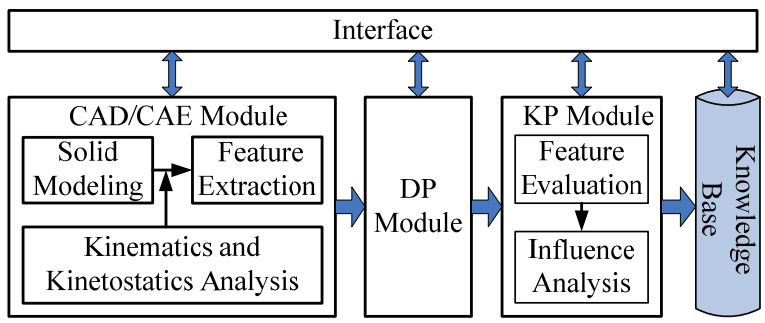

Figure1. FE\&IA Mechanism

CAD/CAE module. This module is constructed to carry out the stress survey for model samples and extract the stress features from samples, which includes three sub-modules, i.e., the solid modeling sub-module based on Pro/Engineering, the kinematics and kinetostatics analysis sub-module, as well as the feature extraction sub-module based on ANSYS. In this module, the efficiency of modeling and analysis can be improved greatly by connecting $\mathrm{VC}++$ with Pro/Engineering and ANSYS.

Data processing (DP) module. In this module, the data of stress survey for all samples can be handled, and the statistics analysis of danger situation for stress features in all samples can be realized.

Knowledge processing (KP) module. KP module is constructed to evaluate the stress features of complex

\footnotetext{
Corresponding author: hhy810608@163.com
} 
component and acquire the absolute influence of structural parameters for every stress feature based on Monte Carlo simulation and Spearman rank correlation analysis method.

These three modules can be operated and contacted each other by the interface, and the results of feature extraction and influence analysis can be saved in knowledge base.

\section{The new strategy of exploring the structural performance for complex component}

\subsection{Feature extraction for complex component}

The complexity of stress distribution is determined by the complexity of component. Feature extraction for the structural performance of complex component has always been a bottleneck problem. In order to explore the stress distribution and extract stress features for the complex component, a new method based on stress survey is proposed, which can be realized automatically by the CAD/CAE module.

Some model samples with different values of structural parameters should be constructed and analyzed. The main steps of feature extraction are shown in Fig. 2 and illustrated as follows.

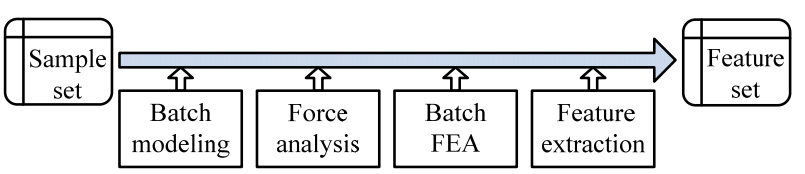

Figure 2. The main steps of feature extraction

In the beginning, some samples are taken by the method of Latin Hypercube Sampling. The value ranges of parameters are determined according to the series components.

- Step1: Batch modeling. The batch modeling of component is realized by the solid modeling sub-module. All the solid model samples must be evaluated and some of geometric deformity models should be taken out.

- Step2: Force analysis. The forces of every model are calculated by the kinematics and kinetostatics analysis sub-module, which will be utilized in the loading of finite element analysis(FEA).

- Step3: Batch FEA. Stress survey based on model samples are carried out by the batch FEA, which are realized by the feature extraction sub-module.

- Step4: Feature extraction. In the post-processing of FEA, each model is divided into several sub-regions and the maximum stress in every sub-region is extracted, which is taken as the feature of stress distribution and called characteristic stress (CS).

In the end, the feature set, i.e., the set saved the CS of all samples, is transmitted to DP module.

\subsection{Influence analysis for complex component}

The influence analysis for complex component is realized by DP module and KP module. The main steps of influence analysis are shown in Fig.3 and illustrated as follows.

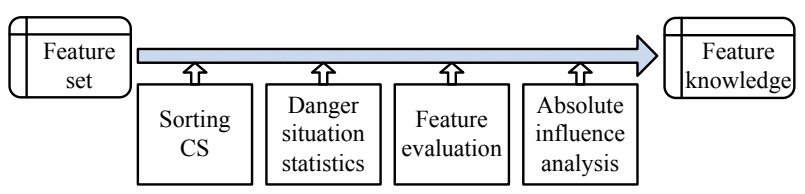

Figure 3. The main steps of influence analysis

-Step1: Sorting CS. After the feature extraction, the feature set can be acquired. For every sample, the CS of all sub-regions should be sorted firstly. According to the result of sorting, the danger regions in every sample can be identified.

-Step2: Danger situation statistics. The danger situation in different sub-region may be different. Therefore, it is necessary to count the times of occurring danger for every sub-region in all samples.

-Step3: Feature evaluation. According to the danger situation, the danger grade of all sub-regions can be identified. And then, the sub-regions can be divided into several classes. The danger sub-region, in which the number of times occurred danger is much higher than other regions, is called feature regions.

- Step4: Absolute influence analysis. In order to extract feature knowledge, the absolute influence analysis for the CS of feature regions is carried out. The Monte Carlo simulation technique and the method based on Spearman rank correlation analysis are utilized in this paper. The Spearman rank correlation models between structure parameters and CS are constructed. The Spearman correlation coefficients are taken as the absolute influence for CS. After that, by using rule reasoning, the feature knowledge is acquired.

\section{Application}

The gooseneck type boom of excavator is taken as an example, in which the structural performance under four working conditions[6] is explored in this paper.

\subsection{Feature extraction for excavator boom}

Firstly, 500 boom samples are generated by the method of Latin Hypercube Sampling, and their solid models are built by the solid modeling sub-module. After evaluating geometry, only 247 boom models are taken as the qualified samples of feature extraction. Secondly, the force analysis for every boom model under four working conditions is achieved, and then the stress survey is carried out.

This stress survey is a comprehensive survey for stress distribution. In order to improve the commonality of the feature extraction sub-module, the sub-regions are acquired by segmenting the line of $\mathrm{BC}$ and the line of $\mathrm{BF}$ equally. In this example, both the line of $\mathrm{BC}$ and the line of $\mathrm{BF}$ are divided into ten equal parts, and the segmentation plane is perpendicular to $\mathrm{BC}$ or $\mathrm{BF}$. According to this division method, a boom model is divided into 23 sub-regions. The division of one of boom sample is shown in Fig. 4 as an example. By using the 
method of feature extraction illustrated in section 3.1, the CS of every sub-region for all boom models are extracted and the feature set is generated.

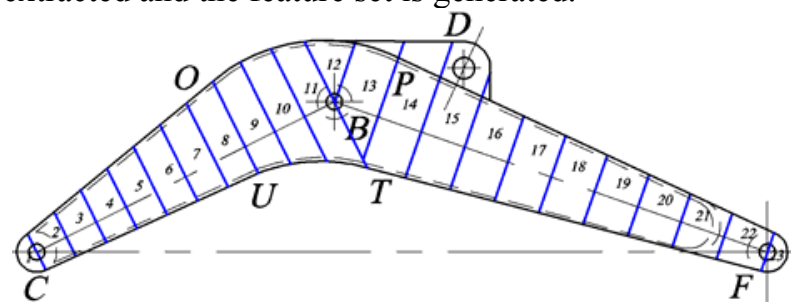

Figure 4. The division of one of boom sample

\subsection{Influence analysis for excavator boom}

After acquiring the feature set, for every sample, the CS of all sub-regions are sorted. And then, the evaluation of danger situation for sub-regions is realized. Danger grades are identified according to the sorting of CS in all sub-regions, i.e., from danger grade 1 to danger grade 23. Danger grade 1 means that the maximum stress in subregion is the global maximum value among 23 subregions. Similarly, danger grade 2 means that the maximum stress in sub-region is the second maximum value among 23 sub-regions, and so on. In this example, the evaluation of danger situation is carried out by counting the times from danger grade 1 to danger grade 5 . The total times of occurring danger for every subregion under four working conditions is shown in Fig.5.

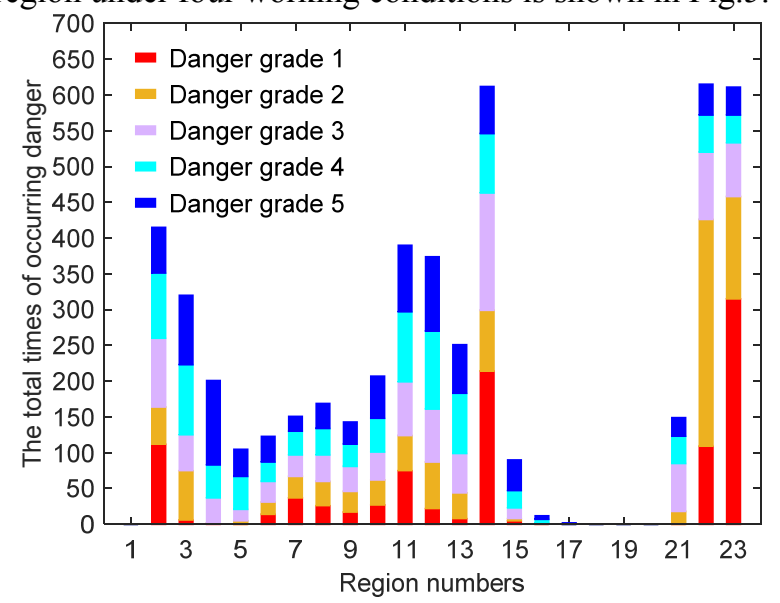

Figure 5. The total times of occurring danger

By this evaluation, the sub-regions can be divided into four classes in this paper, which is shown in Table 1. The four classes are the frequent danger regions, the potential danger regions, the occasional danger regions and the safe regions. Table 1 shows that four regions, i.e., No.2, No.14, No.22 and No.23 region, are the frequent danger regions. The total times of occurring danger in these four regions are significantly higher than other regions. The times of occuring danger in potential danger regions are only second to those in frequent danger regions. Fig.5 also shows that among all potential danger regions, the times of occuring danger in No.11 region is higher than other potential danger regions. In this example, the frequent danger regions and the potential danger regions are taken as feature regions of boom. As the occasional danger regions and the safe regions rarely occur danger, they will no longer be further researched in the following.

Table 1. The classification of sub-regions

\begin{tabular}{|c|c|c|}
\hline Class No. & Name & Region No. \\
\hline Class 1 & Frequent danger & $2,14,22,23$ \\
\hline Class 2 & Potential danger & $6,7,8,9,10,11,12$ \\
\hline Class 3 & Occasional danger & $3,13,15$ \\
\hline Class 4 & Safe & $1,4,5,16,17,18,19,20,21$ \\
\hline
\end{tabular}

Because the values of maximum stress in the frequent danger regions are the most needed to be controlled, the absolute influence of structural parameters for CS in these four regions are analyzed firstly. And the results are shown in the following four figures, i.e., from Fig.6 to Fig.9. The parameters model of boom is shown in Fig.10 and the explanation of structural parameters is shown in Table 2 .

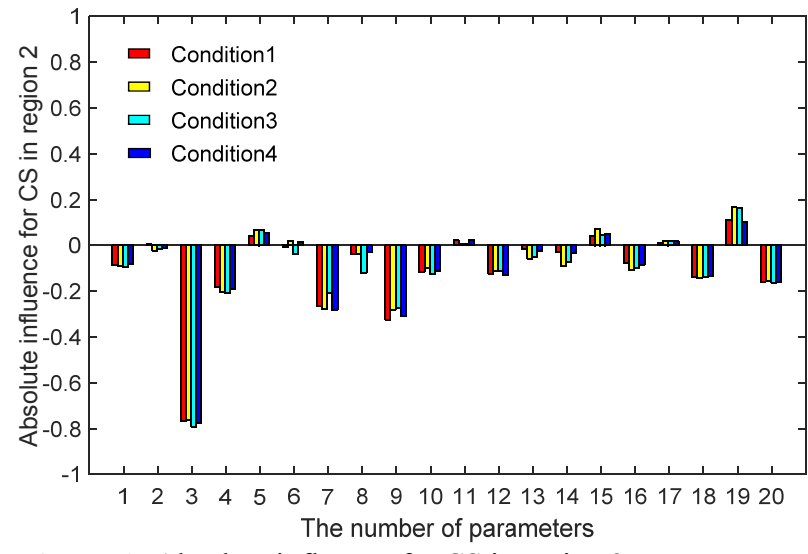

Figure 6. Absolute influence for $\mathrm{CS}$ in region 2

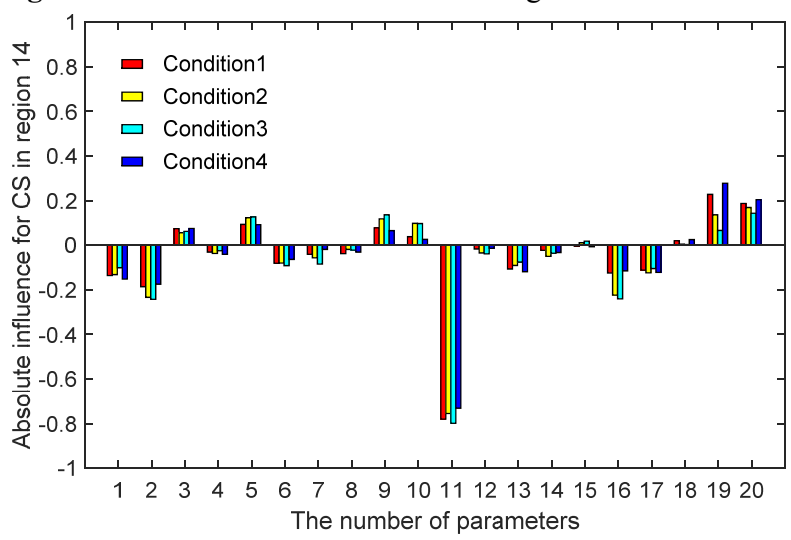

Figure 7. Absolute influence for CS in region 14

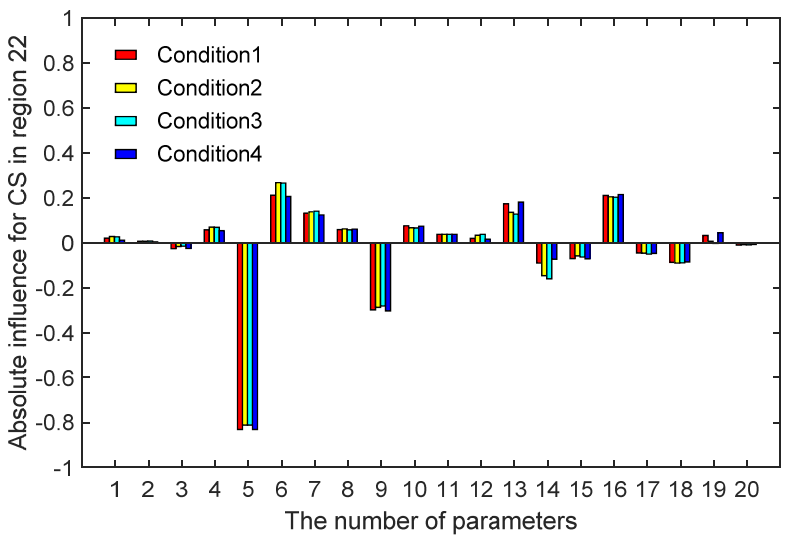

Figure 8. Absolute influence for $\mathrm{CS}$ in region 22 


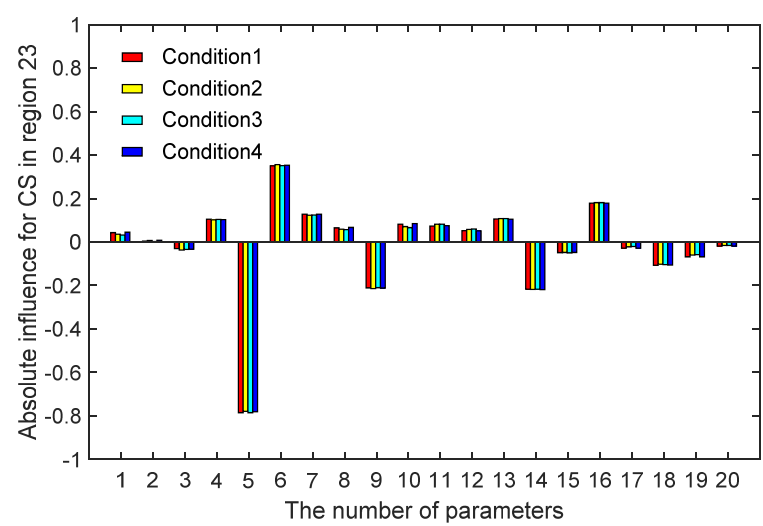

Figure 9. Absolute influence for $\mathrm{CS}$ in region 23

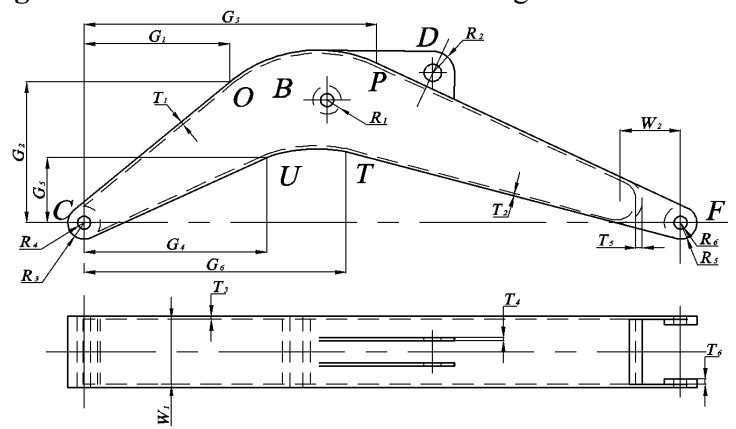

Figure 10. The structural parameters model of boom

Table 2. The explanation of structural parameters

\begin{tabular}{|l|l|l|l|}
\hline NO. & Name & Implication & Type \\
\hline 1 & $\mathrm{R}_{1}$ & External dimeter of reamed hole $\mathrm{B}$ & local \\
\hline 2 & $\mathrm{R}_{2}$ & External dimeter of reamed hole $\mathrm{D}$ & local \\
\hline 3 & $\mathrm{R}_{3}$ & External dimeter of reamed hole $\mathrm{C}$ & local \\
\hline 4 & $\mathrm{R}_{4}$ & Internal dimeter of reamed hole $\mathrm{C}$ & local \\
\hline 5 & $\mathrm{R}_{5}$ & External dimeter of reamed hole $\mathrm{F}$ & local \\
\hline 6 & $\mathrm{R}_{6}$ & Internal dimeter of reamed hole $\mathrm{F}$ & local \\
\hline 7 & $\mathrm{~T}_{1}$ & Thickness of upper cover plate & global \\
\hline 8 & $\mathrm{~T}_{2}$ & Thickness of web plate & global \\
\hline 9 & $\mathrm{~T}_{3}$ & Thickness of side plate & global \\
\hline 10 & $\mathrm{~W}_{1}$ & The distance of side plates & global \\
\hline 11 & $\mathrm{~T}_{4}$ & Thickness of ear plate & local \\
\hline 12 & $\mathrm{~T}_{5}$ & Thickness of bending plate & local \\
\hline 13 & $\mathrm{~W}_{2}$ & $\begin{array}{l}\text { The distance between the center of } \\
\text { bending plate and hole } \mathrm{F}\end{array}$ & local \\
\hline 14 & $\mathrm{~T}_{6}$ & Thickness of lug boss & local \\
\hline 15 & $\mathrm{G}_{1}$ & Lateral distance between $\mathrm{O}$ and $\mathrm{C}$ & geometry \\
\hline 16 & $\mathrm{G}_{2}$ & Portrait distance between $\mathrm{O}$ and $\mathrm{C}$ & geometry \\
\hline 17 & $\mathrm{G}_{3}$ & Lateral distance between $\mathrm{P}$ and $\mathrm{C}$ & geometry \\
\hline 18 & $\mathrm{G}_{4}$ & Lateral distance between $\mathrm{U}$ and $\mathrm{C}$ & geometry \\
\hline 19 & $\mathrm{G}_{5}$ & Portrait distance between $\mathrm{U}$ and $\mathrm{C}$ & geometry \\
\hline 20 & $\mathrm{G}_{6}$ & Lateral distance between T and C & geometry \\
\hline
\end{tabular}

Comparing all of frequent danger regions, the significantly sensitive parameters for CS in No.2, No.14 and No.22 region are different. But the sensitivity of each structural parameter for $\mathrm{CS}$ in No.22 region and No.23 region are similar. Observing the Fig.4 and the results of FEA, it can be found that the danger often occurs in the lug boss, which causes the similar absolute influence for CS in No.22 region and No.23 region. Comparing the absolute influence of each structural parameter for the same CS in Fig.6 to Fig.9, it is shown that the absolute influence of most paremeters under different conditions are similarly. Only a few parameters, such as $\mathrm{G}_{2}, \mathrm{G}_{5}$, the absolute influence under different conditions are relatively different.

According to the absolute influence under four working conditions, the classification of structural parameters for frequent danger regions are realized. For each region, all the structural parameters are divided into four types, i.e., significant sensitive, high sensitive, medium sensitive and low sensitive. The number of parameters in first three classes are shown in Table 3, and the others belong to the low sensititive class are not listed in this paper.

Table 3. The classification of structural parameters for frequent danger regions

\begin{tabular}{|c|c|c|c|}
\hline & $\begin{array}{c}\text { Significant } \\
\text { sensitive }\end{array}$ & $\begin{array}{c}\text { High } \\
\text { sensitive }\end{array}$ & $\begin{array}{c}\text { Medium } \\
\text { sensitive }\end{array}$ \\
\hline No.2 & 3 & 7,9 & $4,19,20$ \\
\hline No.14 & 11 & $2,16,19$ & 20 \\
\hline No.22 & 5 & $6,9,16$ & $7,13,14$ \\
\hline No.23 & 5 & $6,9,14,16$ & 7 \\
\hline
\end{tabular}

Table 3 shows that No.3 parameter, i.e., the external dimeter of reamed hole $\mathrm{C}$, is the significant sensitive parameter for No.2 region; No.11 parameter, i.e., the thickness of ear plate, is the significant sensitive parameter for No. 14 region; and No.5 parameter, i.e., the external dimeter of reamed hole $\mathrm{F}$, is the significant sensitive parameter for No. 22 and No. 23 region. It is indicated that these four regions are the local regions of occurring danger and easily to be controlled, because the significant sensitive parameters for these regions are local parameters of boom.

Table 1 also shows that seven regions, i.e., from No.6 region to No.12 region, are the potential danger regions. Because of the similarly among different conditions, the absolute influence of structural parameters for CS in No.6 region to No.9 region combining four working conditions is shown in Fig. 11.

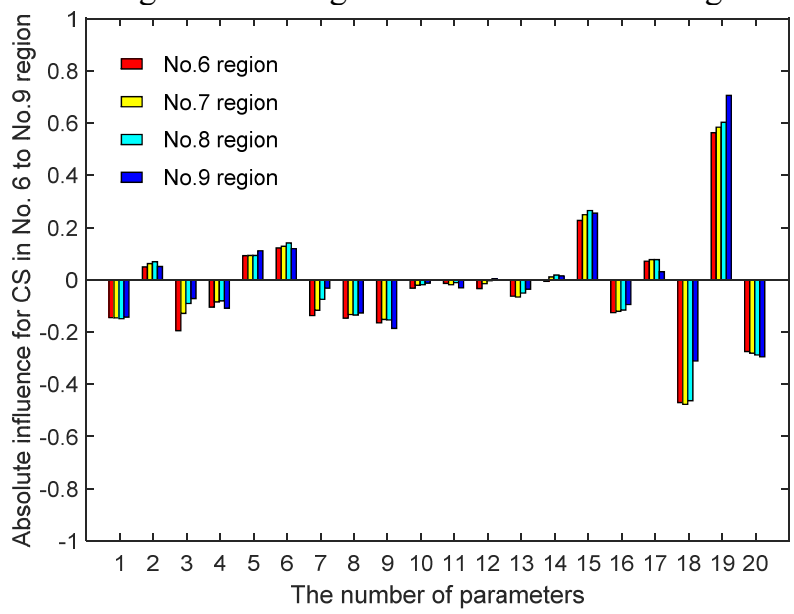

Figure 11. Absolute influence for CS in No.6 region to No.9 region conbining four working conditions

In Fig.11, for most of parameters, the absolute influence of the same parameter for the CS in No.6 to No.9 region are similar. Only a few parameters, such as $\mathrm{R}_{3}, \mathrm{G}_{4}$ and $\mathrm{G}_{5}$, the absolute influence are relatively different.

The absolute influence of structural parameters for CS in No.10 to No.12 region combining four working 
conditions is shown in Fig.12. For many parameters, the absolute influence for the CS in these three regions are different.

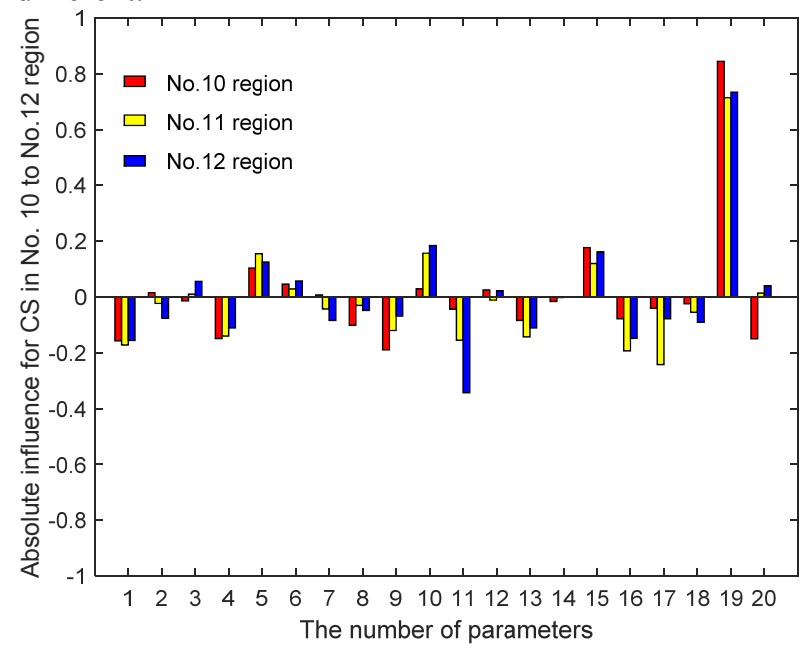

Figure 12. Absolute influence for CS in No.10 region to No.12 region conbining four working conditions

According to Fig.11 and Fig.12, the classification of structural parameters for the regions from No.6 to No.12 is acquired. The number of parameters in three classes are shown in Table 4 and the others belong to the low sensititive class are not listed in this paper.

Table 4. The classification of structural parameters for No. 6 to No. 12 region

\begin{tabular}{|l|l|l|l|}
\hline & $\begin{array}{l}\text { Significant } \\
\text { sensitive }\end{array}$ & $\begin{array}{l}\text { High } \\
\text { sensitive }\end{array}$ & $\begin{array}{l}\text { Medium } \\
\text { sensitive }\end{array}$ \\
\hline No.6 & 18,19 & 15,20 & 3,9 \\
\hline No.7 & 18,19 & 15,20 & 9 \\
\hline No.8 & 18,19 & 15,20 & 9 \\
\hline No.9 & 18,19 & 15,20 & 9 \\
\hline No.10 & 19 & 9 & $1,15,20$ \\
\hline No.11 & 19 & 16,17 & 1,10 \\
\hline No.12 & 11,19 & 10 & $1,15,16$ \\
\hline
\end{tabular}

Table 4 shows that No.18 and No.19 parameters are the significant sensitive parameters for CS in No. 6 to No. 9 region; No. 15 and No.20 parameters are the high sensitive parameters in No. 6 to No. 9 region. It is indicated that these four regions are the global regions of occurring danger and can be controlled by geometry parameters. Table 4 also shows that No.19 parameter is the significant sensitive parameters for CS in No. 10 to No. 12 region. It means that these three regions also can be controlled by geometry parameters. But the influence of global parameters for there three regions is higher than that for No. 6 to No. 9 regions.

\section{Conclusions}

In this paper, a new method of feature extraction and influence analysis for complex component is proposed. By verifying the proposed method in the example of excavator boom, the conclusions are extracted as follows:

(1) The danger regions of complex component may be changed with the change of the values for structural parameters and the working conditions, but it is always concentrated in the certain regions more frequently. It is feasible to take the CS in these regions as the feature of structural performance for complex component.

(2)For each feature region, the influence of every structural paremeter for CS may be different, but at least one significant sensitive or high sensitive parameter can be identified.

(3)According the absolute influence of structural parameters for CS as well as the classification of structural parameters, the controlling parameters for feature regions can be identified. All these result can be utilized to guide the structural optimization of complex component.

\section{Acknowledgements}

This work is supported by the Education Scientific Research Project for the Young Teacher of Fujian Province, China (Grant No. JAT170374), and the Scientific Research Foundation Project of Fujian University of Technology, China (Grant No. GYZ14075).

\section{References}

1. G.H. Shi, Y. Chen, Y.Z. Yang, et al. Journal of Mechanical Engineering. 48,8,(2012),110-114.

2. J. París, F. Navarrina, I. Colominas, et al. Computer Methods in Applied Mechanics and Engineering. 199, (2010), 2110-2122.

3. C.Y. Li, W.C.Wang, Y.Ma. China Mechanical Engineering. 22, 4,(2011), 397-402.

4. C. C. Uzer. Shape optimization of an excavator boom by using genetic algorithm. (Middle East Technical University, Turkey, 2008).

5. D.Q. Zhang, T.X. Zhang, G.S. Zhang, et al. Journal of Mechanical Strength, 33,6, (2011), 913-920.

6. Tianjin Mechanical Engineering Research Institute. GB9141-88, Standardization Administration of the People's Republic of China, (1988). 